\title{
Mass Media Social Marketing Campaigns: A Review
}

\author{
Patrick van Esch ${ }^{1}$, Lisa Rose-Anne Overton ${ }^{2} \&$ Linda Jean van Esch ${ }^{3}$ \\ ${ }^{1}$ Southern Cross Business School, Southern Cross University, Queensland, Australia \\ ${ }^{2}$ Social Policy Research Centre, Middlesex University, London, United Kingdom \\ ${ }^{3}$ Western Australian School of Mines, Faculty of Science \& Engineering, Curtin University, Perth, Australia \\ Correspondence: Patrick van Esch, Southern Cross Business School, Southern Cross University, Locked Mail \\ Bag 4, Coolangatta, QLD 4225, Australia. Tel: 61-7-5589-3043. E-mail: vesch54@ hotmail.com
}

Received: March 18, 2014

Accepted: March 31, 2014

Online Publication: May 27, 2014

doi: 10.5539/ibr.v7n6p1

URL: http://dx.doi.org/10.5539/ibr.v7n6p1

\begin{abstract}
A qualitative data analysis software package NVivo was used to analyse mass media social marketing campaigns to identify if the dimensions of religion were present. The analysis of each campaign included word frequency, a tree map analysis of the campaign as a whole and a tree map analysis of the different campaign mediums. The results indicate that whilst the Material and Narrative or Mythic dimensions had little to no presence across the five (5) campaigns, the following five (5) dimensions were visible across the campaigns: (1) Doctrinal and Philosophical (2) Ethical and Legal (3) Experiential and Emotional (4) Practical and Ritual and (5) Social and Institutional. However, not all of the identifiers within these dimensions were present. The emerging and abstract findings from the textual analysis require further clarification, distilling, elaboration and enhancement. Therefore, a series of questions were developed and in-depth interviews have been recommended to triangulate the findings and add validity when drawing meaningful conclusions.
\end{abstract}

Keywords: dimensions of religion, mass media, social marketing, campaigns, mass media social marketing campaigns, qualitative research, text analysis, NVivo

\section{Introduction}

The analysis and review of mass media social marketing campaigns is a continuation of the body of work outlined in Van Esch et al. (2013) and Van Esch and Van Esch (2013). The Mass media social marketing campaigns (Table 1) were collected from four (4) Australian government and one (1) not-for-profit funded mass media social marketing campaigns that had national distribution (e.g., all Australian states and territories) between the years 2005-2010, where the campaign material was published and easily accessible via the internet. The random sample of campaigns, originate from the following mainstream categories of mass media social marketing campaigns (Van Esch et al., 2013, p. 101): (1) Child abuse (2) Health (3) Skin cancer and (4) Smoking. In terms of the different mass media mediums, the samples included but were not limited to: TV commercials, radio, print (e.g., posters) and outdoors (e.g., bus shelters, shopping trolleys) (Van Esch \& Van Esch, 2013).

Table 1. Summary of mass media social marketing campaigns analysed

\begin{tabular}{cllcccc}
\hline Year & Category & Campaign & TV & Radio & Print & Outdoor \\
\hline 2006 & Health & Get moving & 1 & 4 & 5 & - \\
2007 & Skin cancer & Protect yourself in five ways from skin cancer & 1 & 2 & 3 & - \\
2009 & Child abuse & Adults surviving child abuse & 1 & 6 & 4 & - \\
2009 & Health & Measure up & 2 & 2 & 3 & 4 \\
2010 & Smoking & 4000 chemicals & 1 & 2 & 2 & - \\
\hline
\end{tabular}

Source: Developed for this research.

In support of the relatively small sample size, Van Esch and Van Esch (2013) argue that "in qualitative research studies, there are numerous factors that can determine a sample size and the literature is limited as to what a 
sufficient sample size is (Mason, 2010)". Whilst Guest, Bunce and Johnson (2006, p. 59) argue that "there is little practical guidance for estimating sample sizes for robust research prior to data collection". Therefore, in a review of the literature, seven (7) sources provide practical guidelines for sample sizes (Table 2).

Table 2. Type of research and sample size

\begin{tabular}{lll}
\hline Source & Type of research & Sample size \\
\hline Creswell (1998, p. 64); Morse (1994, p. 225) & Qualitative research & 5 to 25 \\
Bertaux (1981, p. 35) adapted from (Guest et al., 2006) & Qualitative research & $\begin{array}{l}15 \text { is the smallest } \\
\text { acceptable sample }\end{array}$ \\
Charmaz (2006, p. 114) & Qualitative research & 25 is adequate for \\
smaller projects
\end{tabular}

Source: Adapted from Mason (2010); Van Esch and Van Esch (2013).

Using qualitative software (i.e., NVivo 10), the data was then subjected to coding and categorisation in accordance with the research methodology outlined in Van Esch and Van Esch (2013) and an analysis conducted that included:

1) Word frequency;

2) Tree map analysis - Campaign as a whole;

3) Tree map analysis-The different campaign mediums.

\section{Data Analysis and Results}

\subsection{Campaign 1: Health-Get Moving}

The campaign slogan 'get moving, for an hour or more every day' is targeted towards motivating children to spend less time being stationary and more time being active. The results indicate that the slogan appears in both speech and text across all of the different campaign mediums. The consistent use of the slogan is a strong indicator of the practical and ritual dimension through alignment with the ethical insight and preaching identifiers. Preaching the message of conformity in order to bring about redemption through children being healthy is utilised across all of the campaign mediums (e.g., If your children are healthy, you're a good parent). The use of the words 'better get moving' invokes not only preaching but also a sense of motivation through the specific utterance of 'better'.

\subsubsection{Word Frequency}

The campaign was subjected to a word frequency count which analysed words for length, count and weighting (Table 3).

Table 3. Word frequency

\begin{tabular}{lccc}
\hline Word & Length & Count & Weighted Percentage $(\%)$ \\
\hline Get & 3 & 27 & 3.78 \\
Moving & 6 & 23 & 3.22 \\
Sofa & 4 & 16 & 2.24 \\
Day & 3 & 12 & 1.68 \\
Active & 6 & 9 & 1.26 \\
Coverage & 8 & 9 & 1.26 \\
Hour & 4 & 9 & 1.26 \\
Kids & 4 & 9 & 1.26 \\
Children & 8 & 8 & 1.12 \\
\hline
\end{tabular}

Source: Developed for this research. 
The results indicate that only nine (9) words had a weighting $>1 \%$. The two (2) most common words 'get' and 'moving' are directly used in the campaign slogan which is prominent in both speech and text across the different campaign mediums.

\subsubsection{Tree Map Analysis}

The campaign as a whole was subjected to a tree map analysis of the seven (7) dimensions of religion and the identifiers within them (Table 4).

Table 4. Tree map analysis—campaign

\begin{tabular}{|c|c|c|}
\hline Nodes & Coding references & Items coded \\
\hline Practical and RituallPreaching & 26 & 9 \\
\hline Practical and Ritual & 25 & 8 \\
\hline Social and InstitutionallNormative values & 22 & 9 \\
\hline Social and Institutional & 21 & 9 \\
\hline Experiential and Emotional & 19 & 8 \\
\hline Social and InstitutionalIndividual & 18 & 9 \\
\hline Social and InstitutionallGroup & 17 & 9 \\
\hline Social and InstitutionallGroup bonding & 16 & 9 \\
\hline Ethical and Legal & 15 & 7 \\
\hline Ethical and LegallConformity & 15 & 7 \\
\hline Ethical and Legal Judgement of a person & 11 & 6 \\
\hline Experiential and EmotionallEnlightenment & 11 & 7 \\
\hline Experiential and Emotional $\backslash$ Guilt & 9 & 5 \\
\hline Social and InstitutionallCommunity membership & 9 & 7 \\
\hline Social and InstitutionallSymbols\Empty sofa in background & 9 & 4 \\
\hline Experiential and EmotionallSecurity & 8 & 4 \\
\hline Experiential and Emotional\Assurance of salvation & 6 & 5 \\
\hline Experiential and EmotionallLiberation & 6 & 4 \\
\hline Doctrinal and Philosophical\Reflection & 5 & 2 \\
\hline Social and InstitutionallSymbols & 3 & 2 \\
\hline Doctrinal and Philosophical & 2 & 1 \\
\hline Social and InstitutionallFunctioning society & 2 & 2 \\
\hline Social and InstitutionallParticipation in public & 2 & 1 \\
\hline Practical and RituallEthical insight & 1 & 1 \\
\hline Social and InstitutionallFormally organised & 1 & 1 \\
\hline
\end{tabular}

Source: Developed for this research.

The results from the tree map analysis indicate in order of most significant, the dimensions and the identifiers within them. Strong presence:

- Social and Institutional-Community membership, group, group bonding, individual, normative values and symbols.

The following identifiers of the social and institutional dimension whilst still evident had a minor presence: Formally organised, functioning society and participation in public.

Medium presence:

- Doctrinal and Philosophical-Reflection;

- Ethical and Legal — Conformity and judgement of a person; 
- Experiential and Emotional-Assurance of salvation, enlightenment, guilt, liberation, security;

- Practical and Ritual-Preaching.

The following identifiers of the Practical and Ritual dimension whilst still evident had a minor presence: Ethical insight.

Low Presence: There was no evidence of the dimensions and the identifiers within them for-The Material and the Narrative or Mythic.

For further analysis, the different campaign mediums were subjected to a tree map analysis of the seven (7) dimensions of religion and the identifiers as a whole (Table 5).

Table 5. Tree map analysis_-campaign mediums

\begin{tabular}{lcc}
\hline Nodes & Coding references & Items coded \\
\hline Parents 1 radio & 40 & 19 \\
Parent 1 print & 33 & 16 \\
Campaign slogan & 23 & 12 \\
Parent 2 print & 22 & 16 \\
TV commercial & 22 & 15 \\
Radio 2 & 16 & 16 \\
Radio 1 & 15 & 15 \\
Parents 2 radio & 10 & 8 \\
\hline
\end{tabular}

Source: Developed for this research.

The results indicate that symbolism is a key element of the campaign and has a strong presence as an identifier from the Social and Institutional dimension. Specifically, the 'red sofa' is a key symbol and appears in both the poster and TV mediums of the campaign. However, such a strong symbol and its message intent, loses its impact in terms of the radio medium. To overcome the loss of impact, combining the symbol with the Practical and Ritual dimension may help to maintain message intent.

The 'red sofa' is symbolically linked to the Doctrinal and Philosophical dimension and specifically the identifiers reflection and judgement. This is particularly evident towards the end of the TV commercial medium where the sofa appears in the closing scene and a child is about to sit down in front of the television when the sofa skids across the floor into an alternate position so that it is no longer within reach for the child to sit on. With the red sofa having an inference of reflection, the presumption is that the sofa, according to the commercial, is where children spend their time. Then, the TV commercial shows the journey that the children are now active and that the sofa is behind them as a reminder of how far they have come. It also serves as a reminder that a child could 'slip-back' into old unwanted behaviours. Whilst the empty sofa is symbolically powerful in representing reflection and the emerging theme of 'a journey', it is only evident through the visual mediums of the campaign. Symbols might only be powerful or effective visually and may not work within the non-visual (e.g., radio) mediums of the campaign.

There is a medium presence of conformity linked with the 'Ethical and Legal' dimension which is apparent through all the campaign mediums and is directed at parents in the form of judgement. Guilt is often related to judgement in order to persuade conformity. For example: “Want healthy kids? Better get moving”. Whilst the question is rhetorical, parents in general will want healthy children. If the children are not healthy, then the parents are guilty of not 'moving' them. However, the parents are given encouragement through the theme 'assurance of salvation'. For example: "Most kids love being active. They might just need a push in the right direction. And that's where you come in". The message appears to convey the key emergent themes of improvement and the journey to improvement as well as salvation and that the responsibility lies with the parents because they are the leader and in turn the preacher.

\subsection{Campaign 2: Skin Cancer-Protect Yourself in Five Ways from Skin Cancer}

The campaign slogan 'protect yourself in 5 ways from skin cancer' is targeted towards the prevention of skin cancer for all Australians exposed to the sun regardless of age. Linked to the slogan is a set of five (5) symbolic yellow bubbles in a row. The results indicate that the slogan and five (5) symbolic yellow bubbles all appear in 
either speech or text across all of the different campaign mediums. The consistent use of the slogan combined with the yellow bubbles (e.g., Read in the context of the text and hearing the same language) is a strong indicator of the Practical and Ritual dimension through alignment with the preaching and communities re-enacting their myths and stories to confirm and express beliefs through action identifiers.

\subsubsection{Word Frequency}

The campaign was subjected to a word frequency count which analysed words for length, count and weighting (Table 6).

Table 6. Word frequency

\begin{tabular}{|c|c|c|c|}
\hline Word & Length & Count & Weighted Percentage $(\%)$ \\
\hline Cancer & 6 & 13 & 4.41 \\
\hline Skin & 4 & 8 & 2.71 \\
\hline Sunscreen & 9 & 8 & 2.71 \\
\hline Five & 4 & 7 & 2.37 \\
\hline Just & 4 & 7 & 2.37 \\
\hline Melanoma & 8 & 7 & 2.37 \\
\hline Life & 4 & 6 & 2.03 \\
\hline Ways & 4 & 6 & 2.03 \\
\hline Year & 4 & 6 & 2.03 \\
\hline Protect & 7 & 5 & 1.69 \\
\hline Clothing & 8 & 4 & 1.36 \\
\hline Hat & 3 & 4 & 1.36 \\
\hline Outdoors & 8 & 4 & 1.36 \\
\hline Rely & 4 & 4 & 1.36 \\
\hline Shade & 5 & 4 & 1.36 \\
\hline Sunglasses & 10 & 4 & 1.36 \\
\hline Fifteen (15) & 2 & 3 & 1.02 \\
\hline Twenty-two (22) & 2 & 3 & 1.02 \\
\hline Twenty-four (24) & 2 & 3 & 1.02 \\
\hline Advertisement & 13 & 3 & 1.02 \\
\hline $\mathrm{Au}$ & 2 & 3 & 1.02 \\
\hline Australia & 9 & 3 & 1.02 \\
\hline Australian & 10 & 3 & 1.02 \\
\hline Authorised & 10 & 3 & 1.02 \\
\hline Canberra & 8 & 3 & 1.02 \\
\hline Capital & 7 & 3 & 1.02 \\
\hline Common & 6 & 3 & 1.02 \\
\hline Form & 4 & 3 & 1.02 \\
\hline Gov & 3 & 3 & 1.02 \\
\hline Government & 10 & 3 & 1.02 \\
\hline Hell & 4 & 3 & 1.02 \\
\hline Hill & 4 & 3 & 1.02 \\
\hline Mole & 4 & 3 & 1.02 \\
\hline Olds & 4 & 3 & 1.02 \\
\hline Removed & 7 & 3 & 1.02 \\
\hline Skin cancer & 10 & 3 & 1.02 \\
\hline Threatening & 11 & 3 & 1.02 \\
\hline
\end{tabular}

Source: Developed for this research. 
The results indicate that thirty-seven (37) words had a weighting $>1 \%$. The two (2) most common words 'cancer' and 'skin' are directly used in the campaign slogan which is prominent in both speech and text across all of the different campaign mediums. The third most common word 'sunscreen' is one (1) of the yellow bubbles and is either depicted as an image, speech or text across all of the different campaign mediums.

\subsubsection{Tree Map Analysis}

The campaign as a whole was subjected to a tree map analysis of the seven (7) dimensions of religion and the identifiers within them (Table 7).

Table 7. Tree map analysis—campaign

\begin{tabular}{|c|c|c|}
\hline Nodes & Coding references & Items coded \\
\hline Practical and Ritual & 16 & 6 \\
\hline Practical and Ritual|Preaching & 14 & 6 \\
\hline Ethical and Legal & 10 & 5 \\
\hline Ethical and Legal\Conformity & 9 & 5 \\
\hline Experiential and Emotional & 7 & 5 \\
\hline Social and Institutional & 7 & 4 \\
\hline Social and InstitutionalIIndividual & 5 & 4 \\
\hline Experiential and Emotional\Dread & 4 & 4 \\
\hline Social and Institutional 1 Symbols & 4 & 4 \\
\hline Doctrinal and Philosophical & 3 & 3 \\
\hline Social and InstitutionallGroup & 3 & 2 \\
\hline Ethical and LegalUudgement of a person & 2 & 2 \\
\hline Experiential and EmotionallEnlightenment & 2 & 2 \\
\hline Narrative or Mythic & 2 & 2 \\
\hline Narrative or MythiclHuman place in the universe & 2 & 2 \\
\hline Doctrinal and PhilosophicallReflection & 1 & 1 \\
\hline Doctrinal and PhilosophicallReligious narrative & 1 & 1 \\
\hline Doctrinal and PhilosophicallUltimate reality & 1 & 1 \\
\hline Ethical and Legal\A supernatural realm & 1 & 1 \\
\hline Experiential and Emotional\Accumulation & 1 & 1 \\
\hline Practical and RituallEthical insight & 1 & 1 \\
\hline Practical and RituallRe-enactment of myths and stories & 1 & 1 \\
\hline Practical and RituallSpiritual awareness & 1 & 1 \\
\hline Protect yourself in five ways - The five yellow symbols & 1 & 1 \\
\hline Social and InstitutionallCommunity membership & 1 & 1 \\
\hline Social and InstitutionallExemplary individuals & 1 & 1 \\
\hline Social and InstitutionallNormative values & 1 & 1 \\
\hline
\end{tabular}

Source: Developed for this research.

The results from the tree map analysis indicate in order of most significant, the dimensions and the identifiers within them. Strong presence:

- Ethical and Legal—Conformity and judgement.

The following identifier of the Ethical and Legal dimension whilst still evident had a minor presence: A supernatural realm.

- Experiential and Emotional—Dread and enlightenment. 
The following identifier of the Experiential and Emotional dimension whilst still evident had a minor presence: Accumulation.

- Social and Institutional-Exemplary individuals, group community membership, individual, normative values and symbols.

Medium presence:

- Doctrinal and Philosophical—Reflection, religious narrative and ultimate reality;

- Practical and Ritual—Ethical insight, preaching and spiritual awareness.

Low presence:

- Narrative or Mythic-Human place in the universe.

For further analysis, the different campaign mediums were subjected to a tree map analysis of the seven (7) dimensions of religion and the identifiers as a whole (Table 8).

Table 8. Tree map analysis—campaign mediums

\begin{tabular}{lcc}
\hline Sources & Coding references & Items coded \\
\hline Print hell & 35 & 22 \\
Print mole & 21 & 13 \\
Print offend & 16 & 8 \\
TV commercial & 14 & 10 \\
Radio champs & 10 & 9 \\
Radio MCG & 6 & 6 \\
\hline
\end{tabular}

Source: Developed for this research.

The results indicate that the images of the printed mediums (e.g., posters) of the campaign whilst portrayed as clinical and medical, actually have religious undertones with the use of the word 'hell' in text format. Therefore, themes emerge from the mediums which include:

- $\quad$ Life as struggle;

- Life as pain that may or may not be overcome;

- Pain as something that can be prevented if you choose the right rituals;

- Survival.

Furthermore, the target audience is made to feel responsible for their own survival through taking part in the practical application of the five (5) symbolic yellow bubbles. The radio and TV mediums do not use the language of 'hell'. There portrayal of clinical and medical is maintained through images and the message delivered by a professional and subject matter expert in the form of a melanoma surgeon. The Practical and Ritual dimension is evident with the 'Preaching' identifier a strong theme throughout the mediums. Finally, there is an avocation of rituals to encourage salvation from Skin Cancer through taking part in, and practicing the components of the five (5) symbolic yellow bubbles.

\subsection{Campaign 3: Child Abuse-Adults Surviving Child Abuse (ASCA)}

The campaign slogan 'If only it was this easy to get over child abuse. For more than two million Australians it isn't. We can't change their past. Together, we can change their future' is targeted towards adult survivors of child abuse. The results indicate that the slogan appears in either speech or text across all of the different campaign mediums. It provides the context and evokes a strong message, through ironic plays on every-day social and cultural practices.

The printed mediums of the campaign (e.g., posters) are very dark when read without the slogan as context. However, with the slogan printed along the bottom of the poster, it is a strong indicator of the Practical and Ritual and Ethical and Legal dimensions through the alignment with the assurance of salvation, community and hope identifiers. The TV and radio mediums draw explicitly on traditional and social ceremonies. Like the posters, the slogan provides the context to drive the message and ultimately the target audience to a more 
positive place of being. The text highlights a sinister lurking of the 'child abuser' in what should traditionally be a happy moment in a person's life. This invites the target audience to pass judgement on the abuser and empathise with the innocent adult survivor (e.g., victim of suffering). The verbal aspect of the slogan is intended to invoke emotions with the target audience of a promise of hope, peace and salvation for the innocent adult survivor and assurance that the membership of the Adults Surviving Child Abuse (ASCA) community may offer peace.

\subsubsection{Word Frequency}

The campaign was subjected to a word frequency count which analysed words for length, count and weighting (Table 9).

Table 9. Word frequency

\begin{tabular}{lccc}
\hline Word & Length & Count & Weighted Percentage $(\%)$ \\
\hline Abuse & 5 & 18 & 2.99 \\
Child & 5 & 17 & 2.82 \\
ASCA & 4 & 14 & 2.32 \\
Change & 6 & 14 & 2.32 \\
Slogan & 6 & 10 & 1.66 \\
Get & 3 & 7 & 1.16 \\
Image & 5 & 7 & 1.16 \\
Two & 3 & 7 & 1.16 \\
Australians & 11 & 6 & 1.00 \\
Easy & 4 & 6 & 1.00 \\
Future & 6 & 6 & 1.00 \\
Million & 7 & 6 & 1.00 \\
Past & 4 & 6 & 1.00 \\
Print & 5 & 6 & 1.00 \\
\hline
\end{tabular}

Source: Developed for this research.

The results indicate that fourteen (14) words had a weighting $>1 \%$. The two (2) most common words 'abuse' and 'child' are directly used in the campaign slogan which is prominent in both speech and text across all of the different campaign mediums.

\subsubsection{Tree Map Analysis}

The campaign as a whole was subjected to a tree map analysis of the seven (7) dimensions of religion and the identifiers within them (Table 10).

Table 10. Tree map analysis_-campaign

\begin{tabular}{lcc}
\hline Nodes & Coding references & Items coded \\
\hline Ethical and Legal & 16 & 11 \\
Ethical and Legal\Judgement of a person & 16 & 11 \\
Experiential and Emotional & 9 & 7 \\
Practical and Ritual & 9 & 9 \\
Practical and Ritual\Ceremonies & 8 & 8 \\
Experiential and Emotional\Guilt & 6 & 6 \\
Experiential and Emotional\Dread & 4 & 4 \\
Social and Institutional & 4 &
\end{tabular}




\begin{tabular}{|c|c|c|}
\hline Doctrinal and Philosophical & 2 & 2 \\
\hline Doctrinal and PhilosophicallWorldly views and salvation & 2 & 2 \\
\hline Experiential and Emotional\Assurance of salvation & 2 & 2 \\
\hline Experiential and EmotionallComfort & 2 & 2 \\
\hline Experiential and EmotionalLLiberation & 2 & 2 \\
\hline Experiential and EmotionallSecurity & 2 & 2 \\
\hline Practical and RituallEthical insight & 2 & 2 \\
\hline Social and InstitutionallCommunity membership & 2 & 2 \\
\hline Social and Institutional/Group & 2 & 2 \\
\hline Social and InstitutionallGroup bonding & 2 & 2 \\
\hline Social and InstitutionallNormative values & 2 & 2 \\
\hline The Material & 2 & 2 \\
\hline Doctrinal and Philosophical\Reflection & 1 & 1 \\
\hline Experiential and EmotionallEnlightenment & 1 & 1 \\
\hline Experiential and Emotionallnner peace & 1 & 1 \\
\hline Narrative or MythiclThe nature of humans & 1 & 1 \\
\hline Practical and Ritual|Preaching & 1 & 1 \\
\hline Social and InstitutionallFormally organised & 1 & 1 \\
\hline The MateriallSacred areas & 1 & 1 \\
\hline The MateriallSacred places & 1 & 1 \\
\hline
\end{tabular}

Source: Developed for this research.

The results from the tree map analysis indicate in order of most significant, the dimensions and the identifiers within them. Strong presence:

- Practical and Ritual—Ceremonies.

The following identifiers of the Practical and Ritual dimension whilst still evident had a minor presence: Ethical insight and preaching

- Experiential and Emotional—Dread and guilt.

The following identifiers of the Experiential and Emotional dimension whilst still evident had a minor presence: Assurance of salvation, comfort, enlightenment, inner peace, liberation and security:

- Ethical and Legal—Judgement of a person.

- Social and Institutional-Community members, conformity, formally organised, group, group bonding, normative values.

Medium presence:

- Doctrinal and Philosophical-Worldly views and assurance of salvation.

Low Presence:

- Narrative and Mythic - The nature of humanity.

- $\quad$ The Material—Sacred place (e.g., the wedding).

For further analysis, the different campaign mediums were subjected to a tree map analysis of the seven (7) dimensions of religion and the identifiers as a whole (Table 11). 
Table 11. Tree map analysis - Campaign mediums

\begin{tabular}{lcc}
\hline Nodes & Coding references & Items coded \\
\hline Father of the bride TV commercial & 25 & 24 \\
Campaign slogan & 16 & 16 \\
Print card (2) & 9 & 9 \\
Radio 21st birthday (30sec) & 9 & 7 \\
Radio birthday (60sec) & 8 & 4 \\
Radio rugby (30sec) & 8 & 6 \\
Print cake & 7 & 7 \\
Radio birthday (30sec) & 6 & 4 \\
Print beach & 5 & 4 \\
Print T-shirt & 5 & 5 \\
Radio 21st birthday (60sec) & 4 & 4 \\
Radio rugby (60sec) & 2 & 2 \\
\hline
\end{tabular}

Source: Developed for this research.

The results indicate that judgement is a key element of the campaign and has a strong presence as an identifier from the Ethical and Legal dimension. The judgement of a person is directed towards the child abuser, who is not the target-audience of the campaign. The target audience of the campaign is any adult survivor of child abuse. Judgement of the child abuser is encouraged in order to emphasise the innocence of the adult survivor of child abuse. However, the adult survivor of child abuse may still feel guilt even though they are innocent (e.g., as indicated in the slogan). The message invokes the intensity that the guilt is misplaced and can be altered through salvation because the survivor is innocent. Salvation can occur through encouraging the target audience (e.g., adult survivor of child abuse) to join the community membership of the ASCA organisation. A new theme emerges in the printed mediums of the campaign (e.g., posters) that suggests 'shaming' which is strongly linked to guilt. The shaming appears to be directed at the child abuser rather than the adult survivor of child abuse (e.g., target audience).

\subsection{Campaign 4: Health-Measure Up}

The campaign slogan 'How do you measure up?' is targeted towards encouraging male and female adults to decrease their risk of the diseases associated with higher than average waist measurements. The results indicate that the slogan appears in either speech or text across all of the different campaign mediums. The campaign slogan invokes judgement of a person through social norms and is a strong indicator of the Ethical and Legal dimension through alignment with the producers and citizens at every level of existence judge a person identifier. This is further enhanced through the strong use of symbols, particularly the tape measure.

\subsubsection{Word Frequency}

The campaign was subjected to a word frequency count which analysed words for length, count and weighting (Table 12).

Table 12. Word frequency

\begin{tabular}{lccc}
\hline Word & Length & Count & Weighted Percentage (\%) \\
\hline Risk & 4 & 33 & 3.23 \\
Increased & 9 & 25 & 2.44 \\
Measure & 7 & 18 & 1.76 \\
Two (2) & 1 & 17 & 1.66 \\
Health & 6 & 14 & 1.37 \\
Australian & 10 & 11 & 1.08 \\
Day & 3 & 11 & 1.08 \\
Go & 2 & 11 & 1.08 \\
Tape measure & 11 & 11 & 1.08 \\
\hline
\end{tabular}

Source: Developed for this research. 
The results indicate that nine (9) words had a weighting $>1 \%$. The two (2) most common words 'risk' and 'increased' are directly used in all of the print mediums of the campaign. The third (3) most common word 'measure' is directly used in the campaign slogan which is prominent in both speech and text across all of the different campaign mediums.

\subsubsection{Tree Map Analysis}

The campaign as a whole was subjected to a tree map analysis of the seven (7) dimensions of religion and the identifiers within them (Table 13).

Table 13. Tree map analysis_-campaign

\begin{tabular}{|c|c|c|}
\hline Nodes & Coding references & Items coded \\
\hline Ethical and Legal & 52 & 12 \\
\hline Social and Institutional & 44 & 10 \\
\hline Experiential and Emotional & 43 & 10 \\
\hline Ethical and Legal Judgement of a person & 42 & 12 \\
\hline Ethical and Legal\Conformity & 40 & 12 \\
\hline Practical and Ritual & 40 & 12 \\
\hline Practical and Ritual\Preaching & 39 & 11 \\
\hline Social and InstitutionalYndividual & 38 & 9 \\
\hline Social and InstitutionallNormative values & 38 & 10 \\
\hline Experiential and Emotional $\backslash$ Guilt & 30 & 10 \\
\hline Experiential and Emotional\Assurance of salvation & 15 & 8 \\
\hline Social and Institutional\Community membership & 12 & 5 \\
\hline Social and Institutional\Group & 12 & 6 \\
\hline Experiential and EmotionallEnlightenment & 11 & 4 \\
\hline Experiential and Emotional $\backslash$ Comfort & 10 & 5 \\
\hline Experiential and EmotionallLiberation & 10 & 4 \\
\hline Experiential and EmotionallSecurity & 10 & 5 \\
\hline Doctrinal and Philosophical & 7 & 5 \\
\hline Doctrinal and PhilosophicallReflection & 7 & 5 \\
\hline Social and InstitutionallFunctioning society & 5 & 2 \\
\hline Experiential and EmotionallInner peace & 4 & 2 \\
\hline Social and Institutional\Group bonding & 3 & 2 \\
\hline Practical and RituallEthical insight & 2 & 1 \\
\hline Social and InstitutionallFormally organised & 2 & 2 \\
\hline Social and Institutional\Participation in public & 2 & 1 \\
\hline Ethical and LegallSupernatural realm & 1 & 1 \\
\hline Practical and RituallCeremonies & 1 & 1 \\
\hline Social and Institutional/Buildings & 1 & 1 \\
\hline Social and Institutional 1 Symbols & 1 & 1 \\
\hline
\end{tabular}

Source: Developed for this research.

The results from the tree map analysis indicate in order of most significant, the dimensions and the identifiers within them. Strong presence: 
- $\quad$ Social and Institutional-Community membership, individuals, group, normative values.

The following identifiers of the Social and Institutional dimension whilst still evident had a minor presence: Buildings, formally organised, functioning society, group bonding, participation in public and symbols.

- Experiential and Emotional-Assurance of salvation, comfort, enlightenment, guilt, liberation and security.

The following identifier of the Experiential and Emotional dimension whilst still evident had a minor presence: Inner peace.

- $\quad$ Ethical and Legal-Conformity and judgement of a person.

- $\quad$ Practical and Ritual-Preaching.

The following identifiers of the Practical and Ritual dimension whilst still evident had a minor presence: Ceremonies and ethical insight.

Medium presence:

- Doctrinal and Philosophical—Reflection.

Low presence:

There was no presence of either of the following dimensions:

- $\quad$ Narrative or Mythic.

- The Material.

For further analysis, the different campaign mediums were subjected to a tree map analysis of the seven (7) dimensions of religion and the identifiers as a whole (Table 14).

Table 14. Tree map analysis—campaign mediums

\begin{tabular}{lcc}
\hline Nodes & Coding references & Items coded \\
\hline Print male & 98 & 18 \\
TV commercial & 74 & 17 \\
Print more & 69 & 18 \\
Print female & 66 & 21 \\
Outdoor shopping trolley & 57 & 21 \\
Turn around & 52 & 15 \\
Radio 30 second & 28 & 12 \\
Radio 45 second & 27 & 13 \\
Outdoor shopping-center & 22 & 13 \\
Radio 60 second & 13 & 13 \\
Outdoor chronic female & 10 & 5 \\
Outdoor chronic male & 10 & 6 \\
\hline
\end{tabular}

Source: Developed for this research.

The results indicate that judgement is a key element of the campaign and has a strong presence as an identifier from the Ethical and Legal dimension. There are three key symbols evident within both the printed and TV mediums of the campaign:

- Tape measure in the shape of a 'question mark';

- $\quad$ Tape measure in the form of a plank;

- The expanding and shrinking stomach.

The tape measure is a powerful symbol and it is a different way of conceptualising health instead of weight. The tape measure is presented in two (2) different symbolic states which in turn makes the symbolism more powerful as it is presented twice and thus reinforced. It is also a symbol with pre-ascribed meaning in western culture 
where society is encouraged to pay attention to the look and shape of their bodies. Where the tape measure occurs as a 'question mark', judgement of a person is invoked through questioning the target audiences' conformity to normative values. Where the tape measure appears as a plank, the journey from obesity to health is offered (e.g., assurance of salvation).

The 'expanding and shrinking stomach' symbol, like the tape measure symbols, also carries meaning in western culture. The 'fat' stomach is judged and guilt at not conforming is present where one does not conform.

Combined, all three (3) symbols are laden with meaning. These are culturally and socially specific representations of health and body image. The presence of the salvation, judgement and conformity identifiers is a strong indicator of both the Doctrinal and Philosophical and Ethical and Legal dimensions being evident throughout the campaign. For the target audience, those who already occupy a healthy weight are assured of their reduced risk to illness and disease whereas those who do not 'measure up' because of excessive waist lines, are faced with guilt. The terms 'turn around' offers salvation through changing one's lifestyle. This salvation requires a journey and this is an explicit emergent theme.

Within the printed mediums of the campaign (e.g. posters), conformity through judgement of lifestyle is highly evident. The text combined with the images in the posters invoke judgement focusing on the fat around the male or female body's waist and conformity through questioning the target audience's lifestyle with the slogan 'how do you measure up?'. A below the average waist measurement indicates conformity whilst an above the average waist measurement offers the target audience a choice to 'measure up', an opportunity to take action and ultimately become conforming. Across the different campaign mediums, 'preaching language' is less evident. The central theme instead focuses on individual achievement and liberation through conforming to societal values on weight and health. Walking to an unhealthy lifestyle, turning ones back on it and walking towards a healthy lifestyle explicitly indicates a journey and positive transformation.

\subsection{Campaign 5: Smoking - 4000 Chemicals}

The campaign slogan 'Now is the time to quit. Every cigarette is doing you damage' is targeted towards encouraging young Australians aged 18 to 24 years to reject smoking. The results indicate that the slogan appears in either speech or text across all of the different campaign mediums. The 'cigarette' is very symbolic and is used to ascribe negative attributes to the act of smoking to encourage the target audience to see cigarettes as harmful. However, the symbolic nature does not do this alone. The symbolism is combined with the presence of the guilt and judgement identifiers which is a strong indicator of both the Experiential and Emotional and Ethical and Legal dimensions being evident throughout the campaign.

\subsubsection{Word frequency}

The campaign was subjected to a word frequency count which analysed words for length, count and weighting (Table 15).

Table 15. Word frequency

\begin{tabular}{lccc}
\hline Word & Length & Count & Weighted Percentage $(\%)$ \\
\hline Smoking & 7 & 252 & 1.98 \\
One (1) & 1 & 241 & 1.89 \\
NTC & 3 & 217 & 1.70 \\
Three (3) & 1 & 184 & 1.44 \\
Smokers & 7 & 175 & 1.37 \\
Two (2) & 1 & 173 & 1.36 \\
Campaign & 8 & 173 & 1.36 \\
Year: 2010 & 4 & 161 & 1.26 \\
Eighteen (18) & 2 & 129 & 1.01 \\
\hline
\end{tabular}

Source: Developed for this research.

The results indicate that nine (9) words had a weighting $>1 \%$. The most common words 'smoking' are directly used and prominent in both speech and text across all of the different campaign mediums. 


\subsubsection{Tree Map Analysis}

The campaign as a whole was subjected to a tree map analysis of the seven (7) dimensions of religion and the identifiers within them. Table 16 below shows the results.

Table 16. Tree map analysis - campaign

\begin{tabular}{lcc}
\hline Nodes & Coding references & Items coded \\
\hline Ethical and Legal & 11 & 4 \\
Ethical and LegalJudgement of a person & 10 & 4 \\
Practical and Ritual & 10 & 4 \\
Practical and RituallPreaching & 10 & 4 \\
Experiential and Emotional & 8 & 4 \\
Experiential and Emotional\Guilt & 8 & 4 \\
Social and Institutional & 8 & 3 \\
Ethical and LegallConformity & 6 & 4 \\
Social and InstitutionallIndividual & 6 & 3 \\
Experiential and EmotionallDread & 3 & 2 \\
Social and InstitutionallNormative values & 3 & 1 \\
Social and InstitutionallCommunity membership & 2 & 2 \\
Social and InstitutionallGroup & 2 & 1 \\
Social and InstitutionallFunctioning society & 1 & \\
\hline
\end{tabular}

Source: Developed for this research.

The results from the tree map analysis indicate in order of most significant, the dimensions and the identifiers within them. Strong presence:

- Social and Institutional-Community membership, functioning society group, individual and normative values.

- Ethical and Legal-Conformity and judgement of a person.

- Experiential and Emotional—Dread and guilt.

Medium presence:

- $\quad$ Practical and Ritual—Preaching.

Low presence:

- $\quad$ No dimensions or identifiers met this criterion.

For further analysis, the different campaign mediums were subjected to a tree map analysis of the seven (7) dimensions of religion and the identifiers as a whole (Table 17).

Table 17. Tree map analysis—campaign mediums

\begin{tabular}{lcc}
\hline Nodes & Coding references & Items coded \\
\hline Print parent & 34 & 12 \\
TV commercial & 31 & 11 \\
Radio imagine & 12 & 12 \\
Print youth & 11 & 7 \\
\hline
\end{tabular}

Source: Developed for this research. 
The results indicate that judgement is a key element of the campaign and has a strong presence as an identifier from the Ethical and Legal dimension. Overall, the presence of the dimensions of religion and the identifiers is quite low in this campaign. Four (4) of the seven (7) dimensions appear to have an almost equal presence. In terms of the number of mediums that make-up the campaign, it could be considered that they are quite low which in turn may represent the reason for the overall presence of dimensions and identifiers. The campaign mediums encourage the target audience through appealing to them as community members (e.g., smokers or would-be-smokers). This community is not portrayed in a positive way because it goes against or is in opposition to a functioning society with normal values because those who smoke are knowingly causing physical harm to those around them (e.g., children).

Interestingly, only five (5) of the 4000 chemicals in cigarette smoke are listed to highlight the damaging chemicals that are inhaled into the body. The campaign mediums use a 'preaching' context to help deliver and infuse the campaign message. Preaching is a strong indicator of the Practical and Ritual dimension which has a medium presence throughout the campaign. Furthermore, the campaign makes reference to an 'unborn baby' which acts as a symbol as a prediction of the future. The symbolism encourages the target audience to think ahead in terms of the future and dread the effects of smoking, not only to themselves but to their current or future potential family.

\section{Collective Review of All Campaigns}

The Material and Narrative or Mythic dimensions had little to no presence across the five (5) campaigns. Typically, these two (2) dimensions and their indicators are generally more explicit with their religious content whilst in contrast; the five (5) campaigns do not appear to use religious contexts explicitly or consciously. However, the campaign for 'Skin Cancer' represents an anomaly and is an exception to the rule. The campaign slogan explicitly draws on 'a supernatural realm' which is a strong indication of the Ethical and Legal dimension through use of the word 'hell'.

The following five (5) dimensions were visible across the five (5) campaigns:

1) Doctrinal and Philosophical.

2) Ethical and Legal.

3) Experiential and Emotional.

4) Practical and Ritual.

5) Social and Institutional.

However, not all of the identifiers within these dimensions were present. Within the Doctrinal and Philosophical dimension, on some instances, only one identifier is present across some of the campaigns. Of the five (5) dimensions visible within the campaigns, the identifiers relating to each dimension that occurred have been highlighted in bold and the indicators that did not occur are in plain text (Table 18).

Table 18. Identifiers relating to each dimension

\begin{tabular}{ll}
\hline Dimension & Identifiers \\
\hline Doctrinal and philosophical & $\begin{array}{l}\text { Doctrines, systematic formulation of religious teachings and beliefs, sacred texts, the nature of } \\
\text { divinity, ultimate reality, the relationship of humans to an ultimate, real, divinity. Religious } \\
\text { narrate, reflection, structured beliefs beyond the symbolic aspect of myths, faith, and values of a } \\
\text { tradition, worldly views and salvation. }\end{array}$ \\
& Laws, rules, guidelines or behavioural precepts for conduct according to which the \\
Ethical and Legal & community, employees, employers, consumers, producers and citizens at every level of \\
existence judge a person, conformity, a supernatural realm and higher being of a particular faith. & Evoking religious feelings, direct experiences of the divine, visions, enlightenment, security, \\
comperiential and Emotional & inner peace, bliss, assurance of salvation, brilliant emptiness, an expanded sense of identity, \\
& accumulation of religious knowledge and experiences and unconscious, super-conscious and/or \\
& neurologically induced events of a higher reality. \\
& $\begin{array}{l}\text { Practices and rituals of different traditions and cultures, preaching, prayers, ceremonies, } \\
\text { meditation, worship, spiritual awareness, ethical insight, communities re-enacting their myths and } \\
\text { stories to confirm and express beliefs through action. }\end{array}$ \\
\hline
\end{tabular}


Social and Institutional Tradition, belief system, social organisation, shared and implied attitudes practiced by individuals or the group, community membership, participation in public, exemplary individuals, buildings, works of art, cities, symbols, idols, other creations and places of worship, formally organised, a sense of normative values, group bonding, functioning society and a community to live in.

Source: Adapted from Smart (1996); Van Esch and Van Esch (2013).

\subsection{Common Themes Across the Campaigns}

The preaching identifier which is a strong indicator of the Practical and Ritual dimension is the only common theme that is evident throughout all five (5) campaigns. However, it is explicitly evident as a mode of delivering the information within each campaign. Noting that assurance of salvation, conformity and preaching are all closely linked to judgement and together form the synthesis of the emergent theme. Interestingly, an emergent theme is the use of conflicting identifiers to drive the voluntary behaviour change of the target audience. Within the Experiential and Emotional dimension, the identifiers: Assurance of Salvation, comfort, enlightenment and security often occur in line with the use of the conflicting identifier 'guilt'. Using both contrasts seems to create a journey from negative to positive, explicitly always ending the campaign(s) with the 'assurance of salvation'.

\section{Conclusion and Further Research}

Because the sample of campaigns is relatively few in number, the emerging and abstract findings from the textual analysis require further clarification, distilling, elaboration and enhancement to explore and probe more deeply into the following research questions:

1) Are the seven (7) dimensions of religion used or identified within mass media social marketing campaigns?

2) What reception, understanding, openness to consider, do managers of social marketing campaigns have to a clearly articulated description of the application of the dimensions of religion?

Therefore, intentionally using more than one method of analysing data, in-depth interviews will be used to synthesise the data; this approach will allow the individual methods to complement each other and the comparison will be used to triangulate the findings and add validity in drawing meaningful conclusions and implications.

To help set-the-scene, the following questions have been developed for the in-depth interviews:

1) Do the dimensions of religion have application in mass media social marketing campaigns?

2) The campaigns often use a 'preaching' context; do you think this is related to religious practices?

3) Is a preaching context an appropriate driver for mass voluntary behaviour change?

4) Some campaigns utilise a theme of 'Salvation' without using explicit religious language; do you think the campaign would benefit or suffer from the use of religious language?

5) Is a 'Salvation' theme an appropriate driver for mass voluntary behaviour change?

6) Whilst five (5) of the seven (7) dimensions were evident, many of the identifiers were not; would the increased use of the identifiers in an implicit and/or explicit way reinforce a campaign message?

7) Two dimensions that are explicit in religious content were not evident in the campaigns; would this represent a deliberate extent to mitigate the use of 'Religion' in mass media social marketing campaigns?

8) The two non-evident dimensions: The Material and Narrative or Mythic, do they or their identifiers have application within mass media social marketing campaigns?

\section{References}

Bertaux, D. (1981). Biography and society: The life history approach in the social sciences. London: Sage.

Charmaz, K. (2006). Constructing grounded theory: A practical guide through qualitative analysis. Thousand Oaks, CA: Sage.

Creswell, J. W. (1998). Qualitative Inquiry and Research Design: Choosing among five traditions. Thousand Oaks CA: Sage Publications, Inc.

Guest, G., Bunce, A., \& Johnson, L. (2006). How many interviews are enough? An experiment with data saturation and variability. Field Methods, 18(1), 59. http://dx.doi.org/10.1177/1525822x05279903

Morse, J. M. (1994). Designing funded qualitative research. In N. K. Denzin \& Y. S. Lincoln (Eds.), Handbook 
of Qualitative Research (2nd ed.). Thousand Oaks, CA: Sage.

Ritchie, J., Lewis, J., \& Elam, G. (2003). Designing and selecting samples. In J. Ritchie \& J. Lewis (Eds.), Qualitative Research Practice, A guide for Social Science Students and Researchers, 77-108. Thousand Oaks, CA: Sage.

Van Esch, P., \& Van Esch, L. (2013). Justification of a Qualitative Methodology to Investigate the Emerging Concept: The Dimensions of Religion as Underpinning Constructs for Mass Media Social Marketing Campaigns. Journal of Business Theory and Practice, 1(2), 214-243.

Van Esch, P., Van Esch, L., \& Cowley, J. (2013). The Dimensions of Religion as Underpinning Constructs for Mass Media Social Marketing Campaigns: An Emerging Concept. International Journal of Marketing Studies, 5(1), 96-106. http://dx.doi.org/10.5539/ijms.v5n1p96

\section{Copyrights}

Copyright for this article is retained by the author(s), with first publication rights granted to the journal.

This is an open-access article distributed under the terms and conditions of the Creative Commons Attribution license (http://creativecommons.org/licenses/by/3.0/). 